\title{
Electroencephalography Versus Psychometric Tests in Diagnosis of Minimal Hepatic Encephalopathy
}

\author{
Aktham Ismail Alemam ${ }^{\mathrm{a}, \mathrm{b}}$, Mohamed Ahmad Shaaban ${ }^{\mathrm{a}}$, \\ Mohamed Ahmad Abdelhafez ${ }^{\mathrm{a}}$
}

\begin{abstract}
Background: Minimal hepatic encephalopathy (MHE) in patients with liver cirrhosis is defined by the presence of otherwise unexplained cognitive abnormalities, only detectable on psychometric or neurophysiological testing, in the absence of overt hepatic encephalopathy (OHE). The objectives were to study the incidence of MHE in patients with liver cirrhosis, and to compare the sensitivity of the electroencephalography (EEG) versus psychometric hepatic encephalopathy score (PHES) in its diagnosis.
\end{abstract}

Methods: This study was conducted on 50 patients with liver cirrhosis. All patients underwent complete medical and neurological examination, laboratory investigations, abdominal ultrasound, EEG, and PHES involving star construction test, the number connection tests, block design test, the digit symbol test, the line drawing test and the circle dotting test.

Results: The neuropsychiatric symptoms (but not sufficient to diagnose OHE) were present in $40 \%$ of our patients. The psychometric test results were positive in $80 \%$ of them. EEG records showed that $64.7 \%$ of the patients had no slow waves, $23.5 \%$ showed theta waves, $9.8 \%$ showed delta waves, while no patients showed triphasic waves. There was a significant correlation between slow waves in EEG and inattention, amnesia and disturbed thinking $(\mathrm{P}<0.05)$. Also, it was present between psychometric test results and inattention, amnesia, and sleep disturbances $(\mathrm{P}<0.05)$. There was a very significant correlation between psychometric test and Child score $(\mathrm{P}<0.05)$, while it was not present between Child score and slow waves in EEG records $(\mathrm{P}>0.05)$.

Conclusion: The PHES and EEG are important in diagnosis of MHE in patients with liver cirrhosis, but PHES appears to be more sensitive than EEG.

Keywords: Electroencephalography; Psychometry; Hepatic encephalopathy

Manuscript accepted for publication August 24, 2016

${ }^{a}$ Neurology Department, Menoufiya School of Medicine, Egypt ${ }^{b}$ Corresponding Author: Aktham Ismail Alemam, Neurology Department, Menoufiya School of Medicine, Egypt. Email: e_aktham@yahoo.com

doi: http://dx.doi.org/10.14740/jnr391w

\section{Introduction}

Hepatic encephalopathy (HE) is a complex and potentially reversible neuropsychiatric syndrome characterized by symptoms such as somnolence, confusion, asterixis, extrapyramidal rigidity, convulsions and coma [1]. HE is divided into two primary components: minimal hepatic encephalopathy (MHE) and overt hepatic encephalopathy (OHE) [2]. There is an increasing interest in the early detection of the first one [3]. MHE in patients with liver cirrhosis is defined by the presence of otherwise unexplained cognitive abnormalities, only detectable on psychometric or neurophysiological testing, in the absence of OHE [4]. Patients with MHE have a normal neurological examination; however, they may still be symptomatic. These include disturbances in sleep, memory, attention, and concentration [5]. They may also complain of a decrease of psychomotor speed, deficits in visual perception, visuo-spatial orientation and visuo-constructive abilities [6].

It has been well described that MHE has a subtle but negative impact on a patient's motor skills, and the ability to perform complex tasks such as driving [3]. They also complain of significant impairment of daily functioning, such as social interaction, alertness, emotional behavior, work, home management, recreation and pastimes compared with cirrhotic patients who did not have MHE. Treatment with lactulose improved both cognitive functions and quality of life [7]. Also, MHE predicts the development of OHE and is associated with poor survival and some authors suggest that the failure to diagnose this condition could be classified as a medical error [8].

The optimal MHE diagnosis is a matter of debate. The psychometric hepatic encephalopathy score (PHES), which includes five psychometric tests, is the recommended standard for the diagnosis of MHE [9]. PHES is not widely used because psychometric testing is time consuming, difficult, and not standardized. To overcome these problems, computerized psychometric tests recently have been proposed as a simplified tool for MHE detection. A simplified PHES is as good as the PHES in diagnosing MHE and in predicting the occurrence of OHE [10].

The aims of the present study were to study the incidence of MHE in patients with liver cirrhosis, and to compare the sensitivity of the electroencephalography (EEG) versus psy- 
Table 1. Correlation Between Clinical Neuropsychiatric Manifestations and EEG Changes

\begin{tabular}{|c|c|c|c|c|c|c|c|c|c|c|}
\hline \multirow{3}{*}{$\begin{array}{l}\text { Neuropsychiatric } \\
\text { symptoms }\end{array}$} & \multicolumn{8}{|c|}{ EEG slow waves } & \multirow{3}{*}{$\chi^{2}$} & \multirow{3}{*}{ P-value } \\
\hline & \multicolumn{2}{|c|}{ No $(N=33)$} & \multicolumn{2}{|c|}{ Delta $(N=5)$} & \multicolumn{2}{|c|}{ Theta $(\mathrm{N}=12)$} & \multicolumn{2}{|c|}{ Total $(\mathrm{N}=\mathbf{5 0})$} & & \\
\hline & No. & $\%$ & No. & $\%$ & No. & $\%$ & No. & $\%$ & & \\
\hline Attention & & & & & & & & & & $<0.05^{*}$ \\
\hline Normal & 11 & 33.3 & 1 & 20.0 & 1 & 8.3 & 13 & 26.0 & 4.0 & \\
\hline Impaired & 22 & 66.7 & 4 & 80.0 & 11 & 91.7 & 37 & 74.0 & & \\
\hline Memory & & & & & & & & & & $<0.05^{*}$ \\
\hline Normal & 1 & 3 & 2 & 40.0 & 2 & 16.7 & 5 & 10.0 & 7.37 & \\
\hline Impaired & 32 & 97 & 3 & 60.0 & 10 & 83.3 & 45 & 90.0 & & \\
\hline Mood & & & & & & & & & & $>0.05$ \\
\hline Normal & 9 & 27.3 & 3 & 60.0 & 3 & 28.6 & 15 & 30.0 & 2.4 & \\
\hline Impaired & 24 & 72.7 & 2 & 40.0 & 9 & 71.4 & 35 & 70.0 & & \\
\hline Perception & & & & & & & & & & $>0.05$ \\
\hline Normal & 11 & 33.3 & 5 & 41.7 & 5 & 37.5 & 19 & 38.0 & 1.4 & \\
\hline Impaired & 22 & 66.7 & 7 & 58.3 & 9 & 64.3 & 31 & 62.0 & & \\
\hline Thinking & & & & & & & & & & $<0.05$ \\
\hline Normal & 11 & 33.3 & 4 & 80.0 & 5 & 41.7 & 20 & 40.0 & 4.0 & \\
\hline Impaired & 22 & 66.7 & 1 & 20.0 & 7 & 58.3 & 30 & 60.0 & & \\
\hline Visio-spatial & & & & & & & & & & $>0.05$ \\
\hline Normal & 10 & 30.3 & 3 & 60.0 & 4 & 33.3 & 17 & 34.0 & 1.7 & \\
\hline Impaired & 23 & 69.7 & 2 & 40.0 & 8 & 66.7 & 33 & 66.0 & & \\
\hline Insight & & & & & & & & & & $>0.05$ \\
\hline Normal & 11 & 33.3 & 4 & 80.0 & 5 & 41.7 & 20 & 40.0 & 3.9 & \\
\hline Impaired & 22 & 66.7 & 1 & 20.0 & 7 & 58.3 & 30 & 60.0 & & \\
\hline Judgment & & & & & & & & & & $>0.05$ \\
\hline Normal & 11 & 33.3 & 4 & 80.0 & 5 & 41.7 & 20 & 40.0 & 3.9 & \\
\hline Impaired & 22 & 66.7 & 1 & 20.0 & 7 & 58.3 & 30 & 60.0 & & \\
\hline Abstraction & & & & & & & & & & $>0.05$ \\
\hline Normal & 11 & 33.3 & 4 & 80.0 & 5 & 41.7 & 20 & 40.0 & 3.9 & \\
\hline Impaired & 22 & 66.7 & 1 & 20.0 & 7 & 58.3 & 30 & 60.0 & & \\
\hline Sleep & & & & & & & & & & $>0.05$ \\
\hline Normal & 1 & 3.0 & 1 & 20.0 & 0 & 0.0 & 2 & 4.0 & 3.9 & \\
\hline Impaired & 32 & 97.0 & 4 & 80.0 & 12 & 100.0 & 48 & 96.0 & & \\
\hline Motor deficit & & & & & & & & & & $>0.05$ \\
\hline Normal & 28 & 84.8 & 5 & 100.0 & 12 & 100.0 & 45 & 90.0 & 2.8 & \\
\hline Impaired & 5 & 15.2 & 0 & 0.0 & 0 & 0.0 & 5 & 10.0 & & \\
\hline
\end{tabular}

chometric tests in its early diagnosis.

\section{Patients and Methods}

This study was conducted on 50 patients with liver cirrhosis. They were selected from outpatients departments of Internal Medicine Menoufiya University Hospitals during the period from March 2015 to February 2016. They included 40 males and 10 females; their age ranged from 25 to 65 years with a mean age of $50.4 \pm 6.8$ years. The protocol of the study and patient consent policies were reviewed and approved by the medical ethics committees of the hospital.

\section{Inclusion criteria}

All patients have liver cirrhosis as confirmed by medical examination, abdominal ultrasound and positive laboratory results for hepatitis B surface antigens ( $\mathrm{HbsAg}$ ) or hepatitis C virus antibod- 
ies (HcvAb).

\section{Exclusion criteria}

The exclusion criteria included presence of active gastrointestinal bleeding, any active infection including spontaneous bacterial peritonitis, history of neurological or psychiatric disorders other than HE, chronic diarrhea or constipation, intake of any drugs affecting the mental state, or history of cardiopulmonary, renal or endocrinal diseases.

All patients were subjected to the following. 1) Thorough history taking with special emphasis on manifestations of liver cirrhosis, neuropsychiatric symptoms, and precipitating factors of HE. 2) Thorough medical and neurological examination with special emphasis on signs of liver cell failure (e.g. jaundice pallor, ascites, hepatomegaly, splenomegaly, and lower limb edema) and signs of hepatic encephalopathy. 3) Laboratory investigations including complete blood count, renal function tests, and liver function tests involving $\mathrm{HbsAg}$ and HcvAb, serum albumin, total serum bilirubin, prothrombin time and activity, aspartate transaminase (AST) and alanine transaminase (ALT). The Child-Pugh score was used to assess the severity of liver cirrhosis. It depends on five points including the total bilirubin, serum albumin, prothrombin time, ascites and presence of hepatic encephalopathy. 4) Abdominal ultrasound: to help in the diagnosis of cirrhosis and to exclude hepatic focal lesions and liver heterogeneity. 5) EEG: for each patient, EEG was done by using 21-channel computerized machine under standard conditions, hyperventilation for $3-5$ min and photic stimulation. 6) Neuropsychological testing: for quantifying cognitive impairment, by applying the following six tests: star construction test; the number connection test (NCT: number connection test A (NCT-A) and number connection test $\mathrm{B}$ (NCT-B)); the block design test; the digit symbol test (DST); the line drawing test; and the circle dotting test.

\section{Statistical analysis}

Data were collected, tabulated, and statistically analyzed using an IBM personal computer with Statistical Package of Social Science (SPSS) version 20 and Epi Info 2000 programs, where the following statistics were applied: 1) Descriptive statistics, in which quantitative data were presented in the form of mean $(\bar{X})$, standard deviation (SD), and range, and qualitative data were presented in the form numbers and percentages. 2) Analytical statistics: used to find out the possible association between the studied factors and the targeted disease. The used tests of significance included Chi-squared test $\left(\chi^{2}\right)$ and Student's $t$-test (for parametric data), and P-value of less than 0.05 was considered significant.

\section{Results}

The results showed that the neuropsychiatric symptoms (but not sufficient to diagnose OHE) were present in $40 \%$ of our patients with liver cirrhosis, while the psychometric test results were positive in $80 \%$ of them.

EEG records showed that $64.7 \%$ of the patients had no slow waves, $23.5 \%$ showed theta waves, $9.8 \%$ showed delta waves, while no patients showed triphasic waves.

There was a significant correlation between slow waves in EEG and certain clinical symptoms as inattention, amnesia and disturbed thinking $(\mathrm{P}<0.05)$ (Table 1).

There was a significant correlation between psychometric test results and inattention, sleep disturbances and amnesia ( $\mathrm{P}$ $<0.05)$ (Table 2).

There was no significant correlation between the clinical neuropsychiatric manifestations and Child score $(\mathrm{P}>0.05)$ (Table 3).

There was no significant correlation between Child score and slow waves appearance $(\mathrm{P}>0.05)$ (Table 4).

There was a very significant correlation between psychometric test and Child score $(\mathrm{P}<0.05)$, in which the more the Child score was, the more the positive results were (Table 5).

\section{Discussion}

MHE, the mildest form of HE, is characterized by subtle motor and cognitive deficits, and impairs health-related quality of life [11]. Cirrhotic patients with MHE have a normal neurological and mental status by the standards of clinical examination, yet demonstrate quantifiable neuropsychological defects [5].

The term MHE refers to the subtle changes in cognitive functions, electrophysiological parameters, cerebral neurochemical/neurotransmitter homeostasis, cerebral blood flow, metabolism, and fluid homeostasis that can be observed in patients with cirrhosis who have no clinical profile sufficient to diagnose HE [12]. The subtle neurocognitive abnormalities primarily affect attention, speed of information processing, and motor abilities and coordination that are not recognizable on standard neurological examination. These neurocognitive abnormalities are independent of sleep dysfunction or problems with the overall intelligence [8].

The true prevalence of MHE in patients with portal hypertension is unknown. The prevalence of MHE has been reported in as many as $20-84 \%$ of cirrhotic patients, depending on which methods or tools are used and fixed diagnostic cutoffs [13].

Large variations in the prevalence of MHE are related to prior episode of OHE, severity of liver disease, age, presence of esophageal varices, and surgical porto-systemic shunts. Patients who develop MHE are older, more often have alcohol as etiology of cirrhosis, have history of OHE in the past, have more severe liver disease, and more often have esophago-gastric varices [14].

EEG is an excellent tool for diagnosing HE in the research setting. HE is associated with a decreased mean frequency of electrical activity in the brain, and the diagnostic sensitivity for HE of this finding ranges between $43 \%$ and $100 \%$ in published studies [15].

The aims of the present study were to study the incidence of MHE in patients with liver cirrhosis by using psychomet- 
Table 2. Correlation Between Neuropsychiatric Manifestations and Psychometric Tests

\begin{tabular}{|c|c|c|c|c|c|c|c|c|}
\hline \multirow{3}{*}{$\begin{array}{l}\text { Clinical neuropsychiatric } \\
\text { manifestations }\end{array}$} & \multicolumn{6}{|c|}{ Psychometric tests results } & \multirow{3}{*}{$\chi^{2}$} & \multirow{3}{*}{ P-value } \\
\hline & \multicolumn{2}{|c|}{ Positive $(\mathrm{N}=40)$} & \multicolumn{2}{|c|}{ Negative $(\mathrm{N}=10)$} & \multicolumn{2}{|c|}{ Total $(\mathrm{N}=\mathbf{5 0})$} & & \\
\hline & No. & $\%$ & No. & $\%$ & No. & $\%$ & & \\
\hline Attention & & & & & & & & $<0.01 *$ \\
\hline Normal & 3 & 7.5 & 10 & 100.0 & 13 & 26.0 & 35.5 & \\
\hline Impaired & 37 & 92.5 & 0 & 0.0 & 37 & 74.0 & & \\
\hline Memory & & & & & & & & $<0.01 *$ \\
\hline Normal & 2 & 5.0 & 3 & 30.0 & 5 & 10.0 & 5.6 & \\
\hline Impaired & 38 & 95.0 & 7 & 70.0 & 45 & 90.0 & & \\
\hline Mood & & & & & & & & $>0.05$ \\
\hline Normal & 12 & 30.0 & 3 & 30.0 & 15 & 30.0 & 0.00 & \\
\hline Impaired & 28 & 70.0 & 7 & 70.0 & 35 & 70.0 & & \\
\hline Perception & & & & & & & & $>0.05$ \\
\hline Normal & 16 & 40.0 & 3 & 30.0 & 19 & 38.0 & 0.340 & \\
\hline Impaired & 24 & 60.0 & 7 & 70.0 & 31 & 62.0 & & \\
\hline Thinking & & & & & & & & $>0.05$ \\
\hline Normal & 17 & 42.5 & 3 & 30.0 & 20 & 40.0 & 0.521 & \\
\hline Impaired & 23 & 57.5 & 7 & 70.0 & 30 & 60.0 & & \\
\hline Visio-spatial & & & & & & & & $>0.05$ \\
\hline Normal & 14 & 35.0 & 3 & 30.0 & 17 & 34.0 & 0.089 & \\
\hline Impaired & 26 & 65.0 & 7 & 70.0 & 33 & 66.0 & & \\
\hline Insight & & & & & & & & $>0.05$ \\
\hline Normal & 17 & 42.5 & 3 & 30.0 & 20 & 40.0 & 0.521 & \\
\hline Impaired & 23 & 57.5 & 7 & 70.0 & 30 & 60.0 & & \\
\hline Judgment & & & & & & & & $>0.05$ \\
\hline Normal & 17 & 42.5 & 3 & 30.0 & 20 & 40.0 & 0.521 & \\
\hline Impaired & 23 & 57.5 & 7 & 70.0 & 30 & 60.0 & & \\
\hline Abstraction & & & & & & & & $>0.05$ \\
\hline Normal & 17 & 42.5 & 3 & 30.0 & 20 & 40.0 & 0.521 & \\
\hline Impaired & 23 & 57.5 & 7 & 70.0 & 30 & 60.0 & & \\
\hline Sleep & & & & & & & & $<0.05^{*}$ \\
\hline Normal & 0 & 0.0 & 2 & 20.0 & 2 & 4.0 & 8.33 & \\
\hline Impaired & 40 & 100 & 8 & 80.0 & 48 & 96.0 & & \\
\hline Motor deficit & & & & & & & & $>0.05$ \\
\hline Normal & 38 & 5.0 & 7 & 70.0 & 9 & 18.0 & 5.5 & \\
\hline Impaired & 2 & 95.0 & 3 & 30.0 & 41 & 82.0 & & \\
\hline
\end{tabular}

ric tests PHES and EEG, and to know whether PHES or EEG is more sensitive in its diagnosis. The results showed that the neuropsychiatric symptoms (but not sufficient to diagnose OHE) were present in $40 \%$ of our patients. The psychometric tests results were positive in $80 \%$ of them. EEG records showed that $64.7 \%$ of the patients had no slow waves, $23.5 \%$ showed theta waves, $9.8 \%$ showed delta waves, while no patients showed triphasic waves.

These results are in agreement with those of Wunsch et al
[16] who had studied 50 patients with cirrhosis without OHE. PHES had a sensitivity of $57 \%$ and specificity of $97 \%$ to diagnose MHE. In patients with cirrhosis, PHES correlated with severity of liver disease and EEG. In patients with impaired EEG, PHES was lower than in individuals with unaltered EEG; however, agreement between these two modalities was limited.

Also, our results are in the same direction with Duarte-Rojo et al [17] who had enrolled volunteers and patients with cir- 
Table 3. Correlation Between Neuropsychiatric Manifestations and Child Score

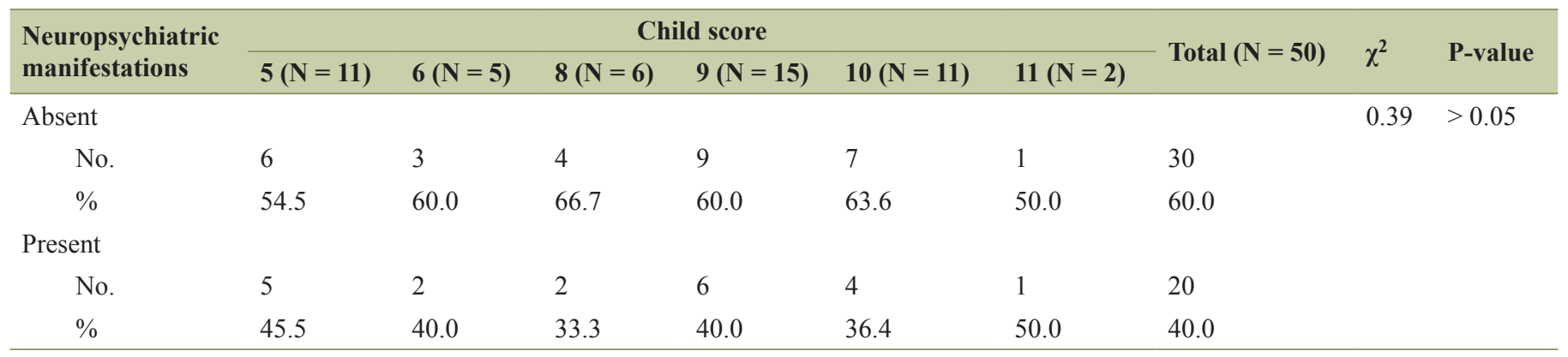

Table 4. Correlation Between EEG Slow Waves and Child Score

\begin{tabular}{|c|c|c|c|c|c|c|c|c|c|}
\hline \multirow{2}{*}{ EEG slow waves } & \multicolumn{6}{|c|}{ Child score } & \multirow{2}{*}{ Total $(\mathbf{N}=\mathbf{5 0})$} & \multirow{2}{*}{$\chi^{2}$} & \multirow{2}{*}{ P-value } \\
\hline & $5(N=11)$ & $6(N=5)$ & $8(N=6)$ & $9(\mathrm{~N}=15)$ & $10(N=11)$ & $11(\mathrm{~N}=2)$ & & & \\
\hline Absent & & & & & & & & 4.63 & $>0.05$ \\
\hline No & 8 & 4 & 4 & 9 & 6 & 2 & 33 & & \\
\hline$\%$ & 72.7 & 80.0 & 66.7 & 60.0 & 54.5 & 100.0 & 66.0 & & \\
\hline \multicolumn{10}{|l|}{ Delta waves } \\
\hline No & 0 & 0 & 1 & 2 & 2 & 0 & 5 & & \\
\hline$\%$ & 0.0 & 0.0 & 16.7 & 13.3 & 18.2 & 0.0 & 10.0 & & \\
\hline \multicolumn{10}{|l|}{ Theta waves } \\
\hline No & 3 & 1 & 1 & 4 & 3 & 0 & 12 & & \\
\hline$\%$ & 27.3 & 20.0 & 16.7 & 26.7 & 27.3 & 0.0 & 24.0 & & \\
\hline
\end{tabular}

rhosis with and without low-grade OHE. Eighty-four patients with cirrhosis lacked evidence of $\mathrm{OHE}$, whereas 20 had OHE. On psychometric testing, 13 of the 84 patients (15\%) with cirrhosis (but without OHE) had MHE.

Li et al [18] applied the five tests of PHES in 146 healthy volunteers. In total, 53 patients with liver cirrhosis completed the PHES. Of the patients with liver cirrhosis had Child-Pugh grades A, B and C, respectively. MHE was diagnosed in 26 patients (49.1\%). Compared with compensated cirrhotic patients (Child A), decompensated cirrhotic patients (Child B and C) had a higher proportion of MHE (65.5\% vs. $29.2 \%)$. NCTA and DST were able to diagnose MHE with a sensitivity of $76.9 \%$ and a specificity of $96.3 \%$. Li et al concluded that PHES is diagnostic to MHE.
Gad et al [19] designed a study to screen for MHE in drivers with liver cirrhosis. A total of 66 patients showed evidence for MHE out of 139 patients who fulfilled the inclusion criteria. No significant differences were present, apart from a significantly elevated arterial ammonia level and a bad self-reported driving history in the MHE-positive group when compared with the MHE-negative group. Multivariate logistic regression revealed that advanced Child-Pugh grade, hepatitis $B$ virus (HBV)-related etiology and smoking are significant risk factors for MHE.

On the other hand, Maric et al [20] found that all patients with cirrhosis had some changes in EEG. Study results showed $80 \%$ of cirrhosis patients to have signs of MHE. Patients were tested using three neuropsychological tests: mini-mental score

Table 5. Correlation Between Psychometric Test and Child Score

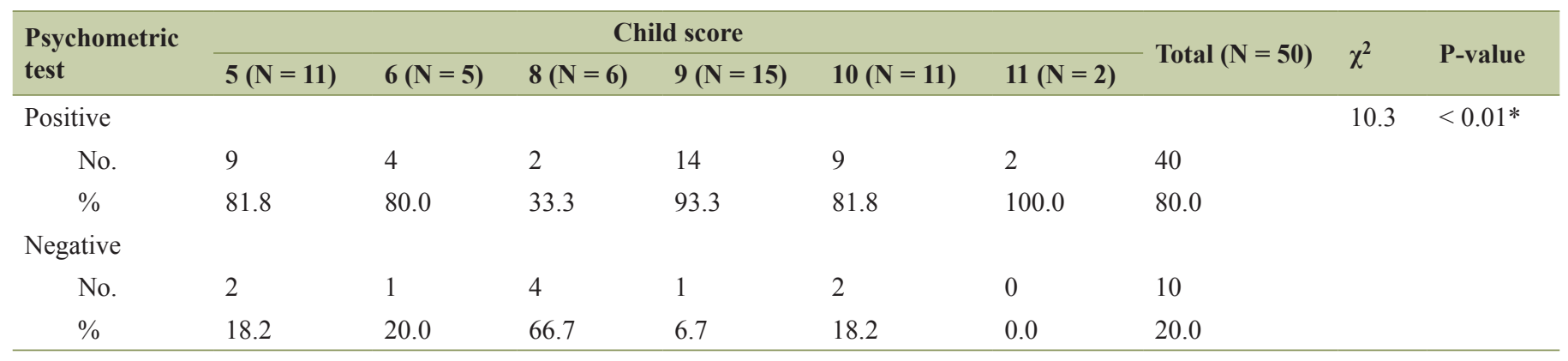


for quick assessment of cognitive status and two tests specific for MHE changes, trail making test part A (TMT-A) and DST. The Child-Pugh score influenced performance on the neuropsychological tests. DST more readily identified patients with MHE. With comparison to our study, these results may be due to use only one test (DST) not all tests of the battery of PHES in the study. So EEG appears to be more accurate than psychometric tests in opposite to our results.

Saxena et al [21] studied 75 non-encephalopathic cirrhotic patients by using a battery for testing the intelligence and memory, the NCT, EEG and auditory P300 event related potentials (P3ERP). Thirty-five out of 75(47\%) patients were diagnosed to have MHE. Nearly $59 \%$ of patients with MHE progressed to overt encephalopathy within a mean duration of 4 months. Multivariate analysis showed that abnormality on EEG and abnormal performance on psychometric battery of tests are among the risk factors for development of overt encephalopathy.

There are many theories to explain pathogenesis of HE. It is believed that neurotoxic substances, including ammonia and manganese, may gain entry into the brain in the setting of liver failure. These neurotoxic substances may then contribute to morphologic changes in astrocytes, and the neuropsychiatric manifestations [22].

It has been shown conclusively that cognitive functions improve with therapy for MHE. Such therapy may improve quality of life of the patients with $\mathrm{MHE}$ and delay the development of OHE [5].

\section{Conclusion}

The PHES and EEG are important in diagnosis of MHE in patients with liver cirrhosis, but PHES appears to be more sensitive than EEG. It may be recommended for all patients with liver cirrhosis to be subjected to testing for MHE. Special attention should be given to those who have cognitive symptoms and high-risk groups such as active drivers, patients handling heavy machines or reporting decline in work performance [23].

\section{References}

1. Bismuth M, Funakoshi N, Cadranel JF, Blanc P. Hepatic encephalopathy: from pathophysiology to therapeutic management. Eur J Gastroenterol Hepatol. 2011;23(1):822.

2. Bajaj JS, Wade JB, Sanyal AJ. Spectrum of neurocognitive impairment in cirrhosis: Implications for the assessment of hepatic encephalopathy. Hepatology. 2009;50(6):20142021.

3. Amodio P, Montagnese S, Gatta A, Morgan MY. Characteristics of minimal hepatic encephalopathy. Metab Brain Dis. 2004;19(3-4):253-267.

4. Sharma P, Sharma BC, Puri V, Sarin SK. Critical flicker frequency: diagnostic tool for minimal hepatic encephalopathy. J Hepatol. 2007;47(1):67-73.

5. Prasad S, Dhiman RK, Duseja A, Chawla YK, Sharma
A, Agarwal R. Lactulose improves cognitive functions and health-related quality of life in patients with cirrhosis who have minimal hepatic encephalopathy. Hepatology. 2007;45(3):549-559.

6. Weissenborn K, Ennen JC, Schomerus H, Ruckert N, Hecker H. Neuropsychological characterization of hepatic encephalopathy. J Hepatol. 2001;34(5):768-773.

7. Weissenborn K. Diagnosis of subclinical hepatic encephalopathy. Med Sci Monit. 2009;5:568-575.

8. Ortiz M, Jacas C, Cordoba J. Minimal hepatic encephalopathy: diagnosis, clinical significance and recommendations. J Hepatol. 2005;42(Suppl 1):S45-53.

9. Randolph C, Hilsabeck R, Kato A, Kharbanda P, Li YY, Mapelli D, Ravdin LD, et al. Neuropsychological assessment of hepatic encephalopathy: ISHEN practice guidelines. Liver Int. 2009;29(5):629-635.

10. Riggio O, Masini A, Efrati C, Nicolao F, Angeloni S, Salvatori FM, Bezzi M, et al. Pharmacological prophylaxis of hepatic encephalopathy after transjugular intrahepatic portosystemic shunt: a randomized controlled study. J Hepatol. 2005;42(5):674-679.

11. Dhiman RK, Chawla YK. Minimal hepatic encephalopathy. Indian J Gastroenterol. 2009;28(1):5-16.

12. Tan HH, Lee GH, Thia KT, Ng HS, Chow WC, Lui HF. Minimal hepatic encephalopathy runs a fluctuating course: results from a three-year prospective cohort follow-up study. Singapore Med J. 2009;50(3):255-260.

13. Lockwood AH, Yap EW, Wong WH. Cerebral ammonia metabolism in patients with severe liver disease and minimal hepatic encephalopathy. J Cereb Blood Flow Metab. 1991;11(2):337-341.

14. Boyer TD, Haskal ZJ. The role of transjugular intrahepatic portosystemic shunt in the management of portal hypertension. Hepatology. 2005;41(2):386-400.

15. Montagnese S, Amodio P, Morgan MY. Methods for diagnosing hepatic encephalopathy in patients with cirrhosis: a multidimensional approach. Metab Brain Dis. 2004;19(3-4):281-312.

16. Wunsch E, Koziarska D, Kotarska K, Nowacki P, Milkiewicz P. Normalization of the psychometric hepatic encephalopathy score in Polish population. A prospective, quantified electroencephalography study. Liver Int. 2013;33(9):1332-1340.

17. Duarte-Rojo A, Estradas J, Hernandez-Ramos R, Poncede-Leon S, Cordoba J, Torre A. Validation of the psychometric hepatic encephalopathy score (PHES) for identifying patients with minimal hepatic encephalopathy. Dig Dis Sci. 2011;56(10):3014-3023.

18. Li SW, Wang K, Yu YQ, Wang HB, Li YH, Xu JM. Psychometric hepatic encephalopathy score for diagnosis of minimal hepatic encephalopathy in China. World J Gastroenterol. 2013;19(46):8745-8751.

19. Gad YZ, Zaher AA, Moussa NH, El-desoky AE, Al-Adarosy HA. Screening for minimal hepatic encephalopathy in asymptomatic drivers with liver cirrhosis. Arab J Gastroenterol. 2011;12(2):58-61.

20. Maric D, Klasnja B, Filipovic D, Brkic S, Ruzic M, Bugarski V. Minimal hepatic encephalopathy in patients with decompensated liver cirrhosis. Acta Clin Croat. 
2011;50(3):375-380.

21. Saxena N, Bhatia M, Joshi YK, Garg PK, Dwivedi SN, Tandon RK. Electrophysiological and neuropsychological tests for the diagnosis of subclinical hepatic encephalopathy and prediction of overt encephalopathy. Liver. 2002;22(3):190-197.
22. Brusilow SW. Hyperammonemic encephalopathy. Medicine (Baltimore). 2002;81(3):240-249.

23. Kircheis G, Wettstein M, Timmermann L, Schnitzler A, Haussinger D. Critical flicker frequency for quantification of low-grade hepatic encephalopathy. Hepatology. 2002;35(2):357-366. 